\title{
SYNTHESIS AND THERMAL PROPERTIES OF TWO NEW DICATIONIC IONIC LIQUIDS
}

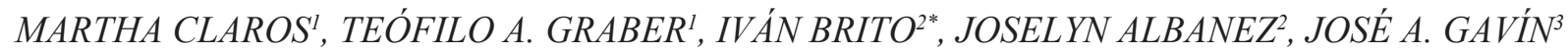

\author{
${ }^{1}$ Departamento de Ingeniería Química, Facultad de Ingeniería, CICITEM, Universidad de Antofagasta, Casilla 170, Av. Angamos 601, Antofagasta-Chile \\ ${ }^{2}$ Departamento de Química, Facultad de Ciencias Básicas, CICITEM, Universidad de Antofagasta, Casilla 170, Av. Angamos 601, Antofagasta-Chile. \\ ${ }^{3}$ Instituto Universitario de Bio-Orgánica “Antonio González”, Universidad de La Laguna, Avda. Astrofísico Francisco Sánchez 2, 38206 \\ La Laguna, Tenerife-España
}

(Received: May 5, 2010 - Accepted: June 9, 2010)

\begin{abstract}
Two new dicationic ionic liquids 1,7-bis(3-methylimidazolium-1-yl)heptane bromide (I) and 1,8-bis(3-methylimidazolium-1-yl)octane bromide (II) have been synthesized and characterized by elemental analysis, FT-IR, ${ }^{1} \mathrm{H}$ and ${ }^{13} \mathrm{C}$ NMR, and TG-DSC techniques. The compound (I) is liquid at room temperature, whereas (II) is liquid at $42.7^{\circ} \mathrm{C}$.
\end{abstract}

Keywords: Dicationic Ionic liquids, thermal properties, synthesis.

\section{INTRODUCTION}

Ionic liquids (ILs) have been recognized as potential new green alternatives to conventional organic solvents in a wide range of synthetic, catalytic, separation and electrochemical applications. They are characterized by their unique physicochemical properties, including low melting points, low inflammability, and tunable hydrophobicities. They are stable in a wide range of temperature, and have great chemical and electrochemical stability. In addition, they have negligible vapor pressures so there is no loss of solvent through evaporation, avoiding environmental problems due to volatilization ${ }^{1-5}$.

The dicationic ionic liquids contain two head groups, linked by a rigid or flexible spacer ${ }^{6}$. This kind of ILs demonstrate unique features in critical micelle concentration, better behavior as lubricants, electrolytes characteristics, surface tension, and higher thermal stabilities than monocationic ILs and other traditional solvents ${ }^{6-9}$. Besides the combination cation-anion, simple changes in the length of the spacer or in the aliphatic chains on the cations, allows the physical properties of these ionic liquids to be tailored for specific applications ${ }^{11-13}$.

We report here, the synthesis, characterization and thermal properties of two new dicationic ionic liquids. The general molecular structure of these compounds is shown in figure 1 .

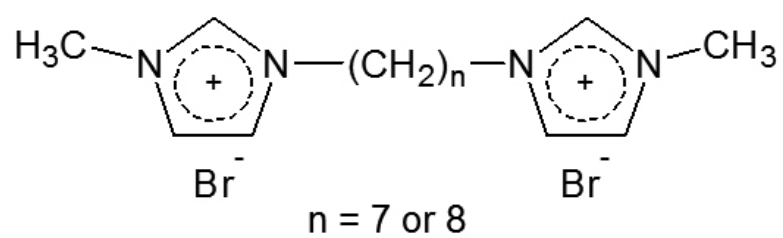

Figure 1: Structure of the new synthesized dicationic ionic liquids.

\section{EXPERIMENTAL}

All materials were purchased from Sigma-Aldrich and used without any additional purification.

NMR experiments were carried out on a Bruker Avance 600 spectrometer operating at $600.23 \mathrm{MHz}$. NMR spectra were recorded at $27^{\circ} \mathrm{C}$ with the HDO signal used as an internal reference $\left(\delta 4.70\right.$ at $\left.27^{\circ} \mathrm{C}\right)$. The compounds were dissolved in $500 \mu \mathrm{L}\left(90 \% \mathrm{H}_{2} \mathrm{O} / 10 \% \mathrm{D}_{2} \mathrm{O}\right)$. Watergate pulse sequences ${ }^{10}$ were used to suppress the proton signal of the solvent. FT-IR was recorded on a Nicolet Avatar 330 spectrophotometer using $\mathrm{KBr}$ pellets. Elemental analysis $(\mathrm{C}, \mathrm{H}, \mathrm{N})$ was performed on a Carlo Erba EA 1108 instrument and the mass spectra were recorded on a VG AutoSpec FISON spectrometer.

Dynamic thermogravimetric measurements were performed using a Mettler TG/SDTA $851^{\mathrm{e}}$ thermobalance. The thermogravimetric results were processed by Mettler calorimetric system using the Start ${ }^{\mathrm{e}}$ program system. Samples were heated in $\mathrm{Al}_{2} \mathrm{O}_{3}$ pans. Measurements were carried out between $0^{\circ} \mathrm{C}$ and $900^{\circ} \mathrm{C}$ at $20^{\circ} \mathrm{min}^{-1}$ under $\mathrm{N}_{2}$.

The calorimetric measurements DSC reported were carried out at a $20 \mathrm{~K} /$ min heating rate in a Mettler 821 calorimetric system, using Start ${ }^{\mathrm{e}}$ program system; the temperature scale was calibrated using indium. The typical amount of sample was $20 \mathrm{mg}$.

The new compounds were synthesized by the following process: 1,7-bis(3- methylimidazolium-1-yl)heptane bromide (I). A mixture of 1 -methylimidazole $(0.25 \mathrm{~mol})$ and 1,7 dibromoheptane $(0.05 \mathrm{~mol})$ were heated at $80^{\circ} \mathrm{C}$ and stirred for $48 \mathrm{~h}$ under dry argon atmosphere. After a slow cooling of the reaction system to room temperature, a yellow oil was separated and washed three times with acetone and then dried under vacuum. Compound (I) was obtained as a viscous yellow oil, with $62.1 \%$ of yield. ${ }^{1} \mathrm{H}$ NMR: $\delta=$ $8.61(\mathrm{H}-2), 7.37(\mathrm{H}-1), 7.32(\mathrm{H}-3), 4.08(\mathrm{H}-4), 3.78(\mathrm{H}-5), 1.74(\mathrm{H}-6), 1.25$ $(\mathrm{H}-7), 1.12(\mathrm{H}-8)$, Fig. $4 .{ }^{13} \mathrm{C}$ NMR: $\delta=135.7(\mathrm{C}-2), 123.5(\mathrm{C}-1), 122.1(\mathrm{C}-$ 3), 49.4 (C-4), 35.7 (C-5), 29.1 (C-6), 27.5 (C-7), 25.1 (C-8). IR (400-4000 $\mathrm{cm}^{-1}$, pellet $\left.\mathrm{KBr}\right): 3087[\mathrm{v}(\mathrm{Ar}-\mathrm{H})] ; 2859[\mathrm{v}(\mathrm{C}-\mathrm{H})] ; 1463[\mathrm{v}(\mathrm{C}=\mathrm{N})] ; 1168$ [u(C-N)]; $1573[\mathrm{v}(\mathrm{C}=\mathrm{C})]$. Elemental analysis calculated $(\%)$ for $\mathrm{C}_{15} \mathrm{H}_{30} \mathrm{~N}_{4} \mathrm{Br}_{2}$ $\cdot \mathrm{H}_{2} \mathrm{O}$ : C 40.93; H 6.41; N 12.73. Found: C 41.31; H 6.89; N 13.16. 1,8-bis(3methylimidazolium-1-yl)octane bromide (II), it was synthesized by similar procedure described above and was obtained as a white solid. Yield $93.3 \% .{ }^{1} \mathrm{H}$ NMR: $\delta=8.59(\mathrm{H}-2), 7.35(\mathrm{H}-1), 7.31(\mathrm{H}-3), 4.06(\mathrm{H}-4), 3.77(\mathrm{H}-5), 1.73(\mathrm{H}-$ 6), 1.18 (H-7, H-8), Fig. 5. ${ }^{13} \mathrm{C}$ NMR: $\delta=135.7$ (C-2), 123.4 (C-1), 122.1 (C-3), 49.5 (C-4), 35.6 (C-5), 29.1 (C-6), 27.8 (C-7)*, $25.2(\mathrm{C}-8)^{*}{ }^{*}$ exchangeable. IR (400-4000 $\mathrm{cm}^{-1}$, pellet $\left.\mathrm{KBr}\right): 3141$ [v(Ar-H)]; 2930 [v(C-H)]; 1463 [v(C=N)]; $1167[\mathrm{v}(\mathrm{C}-\mathrm{N})]$. Elemental analysis calculated (\%) for $\mathrm{C}_{16} \mathrm{H}_{28} \mathrm{~N}_{4} \mathrm{Br}_{2} \cdot \mathrm{H}_{2} \mathrm{O}$ : C 43.66; H 7.33; N 12.73. Found: C 42.31; H 6.66; N 12.33.

\section{RESULTS AND DISCUSSION}

Thermogravimetric (TG) analysis experiments were conducted to determine the thermal stabilities of the novel ILs. As shown in Figure 2, the decomposition temperature $\left(\mathrm{T}_{\mathrm{d}}\right)$ for the sample (I) is $343^{\circ} \mathrm{C}$ and the $\mathrm{T}_{\mathrm{d}}$ for sample (II) is $338^{\circ} \mathrm{C}$, it is clearly that 1,7 -bis(3-methylimidazolium-1-yl)heptane bromide has better thermal stability than 1,8-bis(3-methylimidazolium-1-yl) octane bromide.

The TG curves of (I) and (II), shown in Figure 2, shows a single weight loss for both compounds between 25 and $1300^{\circ} \mathrm{C}$ of $4.6 \%$ and $4.8 \%$ of the original weight, corresponding to the loss of one water molecule for (I) and (II). In both compounds, the TG traces decreased abruptly around $330^{\circ} \mathrm{C}$ and above, corresponding to evaporation for (I) and sublimation in case of (II), this also means the start of decomposition.

Information about thermal stabilities of dicationic ionic liquids is scarce, except for some studies ${ }^{11,14,18,19}$. Generally, thermal stabilities of dicationic ionic liquids are significantly higher than their monocationic analogues due to their higher charge, higher molecular weight, and greater intermolecular interactions 18 . 
Literature reports indicate that the thermal decomposition is strongly dependent on salt structure. Normally in monocationic ionic liquids, increasing the substitution alkyl chain of the imidazolium cation, increases the thermal stability ${ }^{17}$. However, it seems that for dicationic ionic liquids the length of the linkage chain increase the thermal stability ${ }^{14}$. Although molecules of water were found in this work, comparing with a previous report, where the substitution alkyl chain is very long, but the linkage chain is short (1,4-bis(3tetradecylimidazolium-1-yl) butane bromide ${ }^{8}$ ), the decomposition temperature found is $303.5^{\circ} \mathrm{C}$, and in this work the decomposition temperatures are significantly higher, 338 and $343^{\circ} \mathrm{C}$ for (I) and (II) respectively.

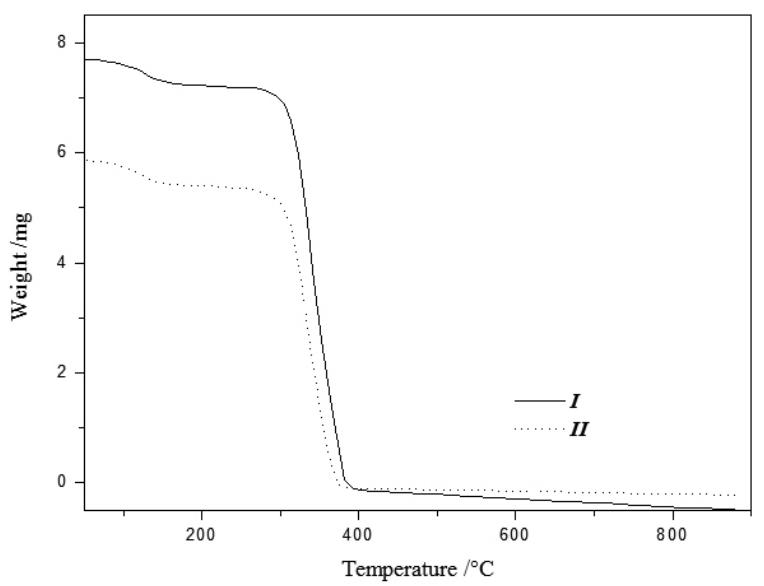

Figure 2: Thermogravimetric curve for Ionic Liquids (I) and (II).

The DSC heating and cooling curves for compound (I) and (II) are illustrated in Figures $3 \mathrm{a}$ and $3 \mathrm{~b}$, respectively. In heating, the compound (I) present two distinct exothermic transitions, an exotherm crystallization temperature $\left(\mathrm{T}_{c}^{h}\right)$ preceding the melting temperature $\left(\mathrm{T}_{s}^{h}\right)$. In the cooling curve, there is a single peak corresponding to a solid-solid transition $\left(\mathrm{T}_{\mathrm{s-s}}{ }^{\mathrm{c}}\right)$. The compound (II) shows a solid-solid transition $\left(\mathrm{T}_{\mathrm{s-s}}{ }^{c}\right)$ before the melting point $\left(\mathrm{T}_{\mathrm{s-}}\right.$ $\left.{ }_{1}^{h}\right)$. In the cooling scan there is a solid-solid transition $\left(\mathrm{T}_{\mathrm{ss}-\mathrm{s}}{ }^{c}\right)$, which is displaced of the heating transition implying an amorphous compound.

In addition, enthalpies for each thermal transition were calculated, and are shown in Table 1.

Table 1: Phase transitions and enthalpies.

\begin{tabular}{|c|c|c|c|c|c|}
\hline \multicolumn{3}{|c|}{ Ionic Liquid I } & \multicolumn{3}{c|}{ Ionic Liquid II } \\
\hline & $\mathrm{T} /\left[{ }^{\circ} \mathrm{C}\right]$ & $\Delta \mathrm{H} /[\mathrm{J} / \mathrm{mol}]$ & & $\mathrm{T} /\left[{ }^{\circ} \mathrm{C}\right]$ & $\begin{array}{c}\Delta \mathrm{H} /[\mathrm{J} / \\
\mathrm{mol}]\end{array}$ \\
\hline $\mathrm{T}_{\mathrm{c}}{ }^{h}$ & 3.03 & $-8.17 \mathrm{E}-3$ & $\mathrm{~T}_{\mathrm{s}-\mathrm{s}}{ }^{h}$ & 14.67 & $1.36 \mathrm{E}-2$ \\
\hline $\mathrm{T}_{\mathrm{s}-1}{ }^{h}$ & 22.94 & $7.40 \mathrm{E}-3$ & $\mathrm{~T}_{\mathrm{s}-1}{ }^{h}$ & 42.67 & $2.50 \mathrm{E}-2$ \\
\hline $\mathrm{T}_{\mathrm{s}-\mathrm{s}}{ }^{c}$ & 3.8 & $4.77 \mathrm{E}-4$ & $\mathrm{~T}_{\mathrm{s}-\mathrm{s}}{ }^{c}$ & -4 & $7.70 \mathrm{E}-3$ \\
\hline
\end{tabular}

\section{${ }^{h}$ Heating \\ ${ }^{c}$ Cooling}

Both compounds had a $T_{s-l}$ below $100^{\circ} \mathrm{C}$, which could be considered as ILs. For (I) the solid-liquid transitions occurs at $22.9^{\circ} \mathrm{C}$, being liquid at room temperature, however (II) has a solid-liquid transition at $42.7^{\circ} \mathrm{C}$. This behavior is contrary to what was expected, since increasing the link chain between the cations, the solid-liquid transition should decrease ${ }^{12,14}$. This phenomenon could be related to the water molecules present in both products.
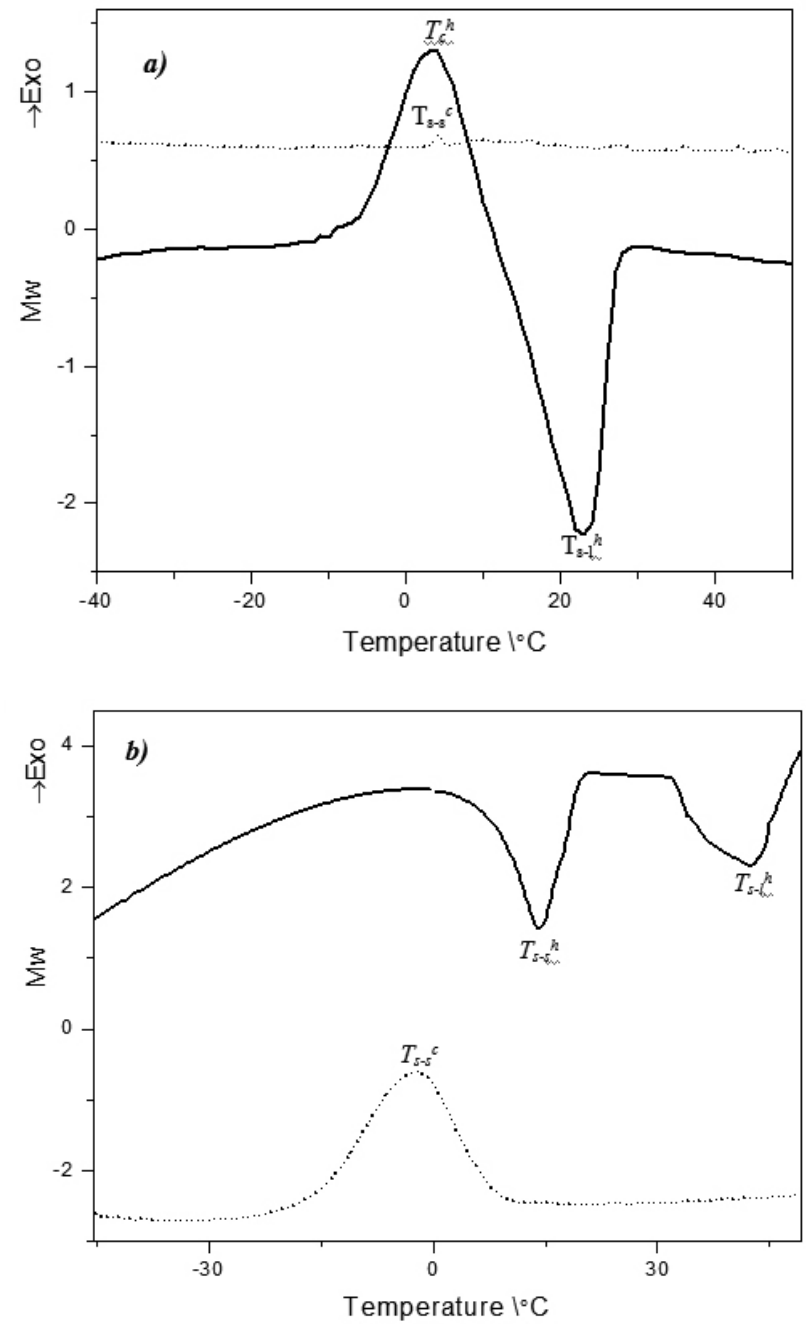

Figure 3: Differential Scanning Calorimetry curves, a) Ionic Liquid (I), b) Ionic Liquid (II)

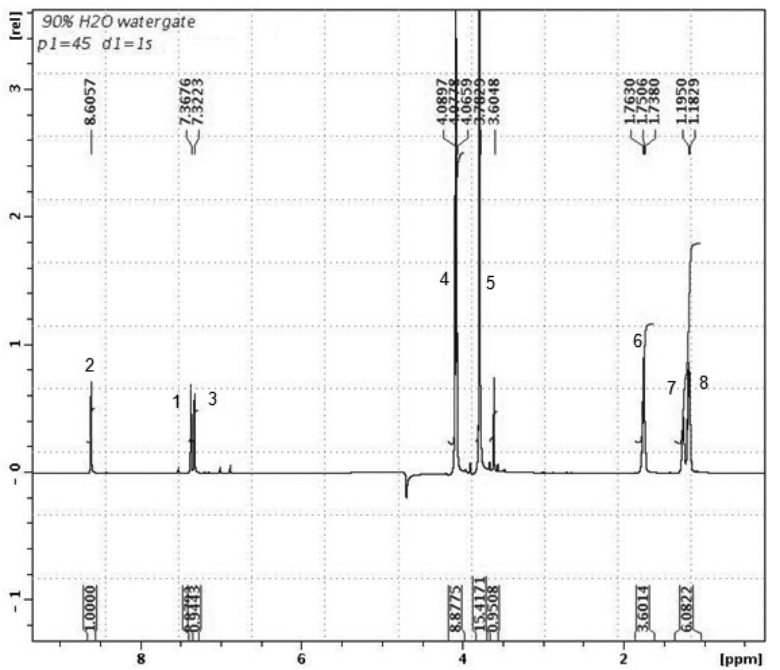




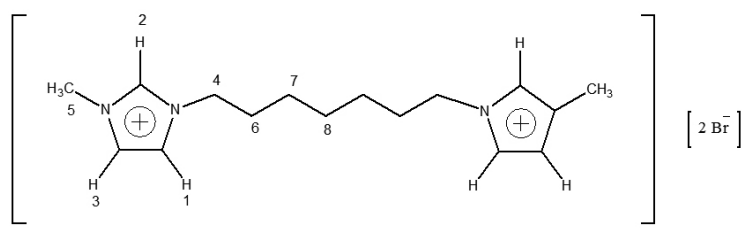
(I).

Figure 4: 1H NMR, 1,7-bis(3-methylimidazolium-1-yl)heptane bromide
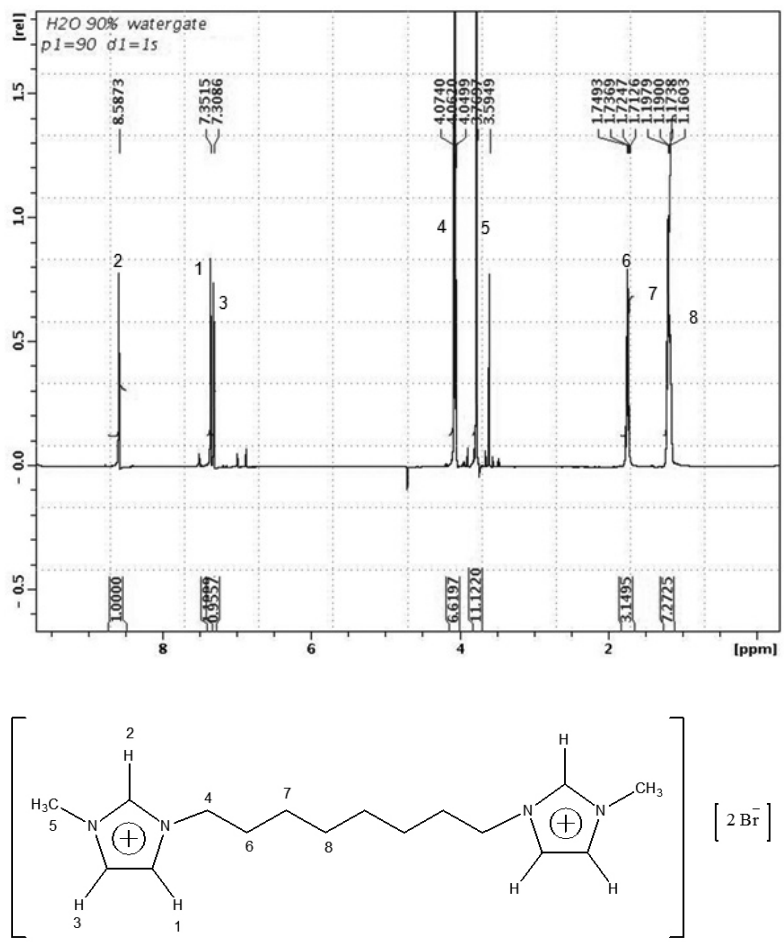
(II).

Figure 5: 1H NMR, 1,8-bis(3- methylimidazolium-1-yl)octane bromide

\section{CONCLUSIONS}

In summary, we presented the synthesis of two new dicationic ionic liquids, 1,7-bis(3-methylimidazolium-1-yl)heptane bromide and 1,8-bis(3methylimidazolium-1-yl)octane bromide, with a simple procedure and obtaining products with high yields. In addition, a complete spectroscopic and thermal analysis was made for both compounds. Both compounds show the presence of water molecules, but this solvent only change slightly the thermal properties of the new ionic liquids reported here, with respect to related unsolvated ILs.

\section{ACKNOWLEDGEMENTS}

This work was supported by the project Fondecyt 1085059 and CICITEM. M. Claros gratefully acknowledge the grant of the Universidad de Antofagasta.

\section{REFERENCES}

1. K. R. Seddon, J. Chem. Tech. Biotechnol., 68, 351-356, (1997).

2. T. Welton, Chem. Rev. 99, 2071-2083 (1999).

3. J.D. Holbrey and K.R. Seddon, J. Chem. Soc., Dalton Trans., 2133-2139, (1999).

4. J. M. Earle and K. R. Seddon, Pure Appl. Chem., 72, 1391-1398, (2000).

5. P. Wasserscheid and T. Welton (Eds.), Ionic Liquids in synthesis, WileyVCH Verlag GmbH \& Co. KGaA, (2002).

6. J. Young Kim, T. Ho Kim, D. Young Kim, N. Park and K. Ahn, Journal of Power Sources, 175, 692-697, (2008).

7. M. Ao, G. Xu, Y. Zhu and Y. Bai, Journal of Colloid and Interface Science, 326, 490-495, (2008).

8. Y.S. Ding, M. Zha, J. Zhang and S.S. Wang, Colloids and Surfaces A: Physicochem. Eng. Aspects, 298, 201-205, (2007).

9. X. Han and D. W. Armstrong, Org. Lett., 7 (19), 4205-4208 (2005).

10. M. Piotto, V. Saudek, and V. Sklenar, J. Biomol. NMR, 2, 661-665 (1992).

11. G. Yu, S. Yan, F. Zhou, X. Liu, W. Liu and Y. Liang, Tribology Letters, 25, 197-205 (2007).

12. Z. Zhang, L. Yang, S. Luo, M. Tian, K. Tachibana and K. Kamijima, Journal of Power Sources, 167, 217-222, (2007).

13. Q. Q. Baltazar, J. Chandawalla, K. Sawyer and J.L. Anderson, Colloids and Surfaces A: Physicochem. Eng. Aspects., 302, 150-156 (2007).

14. J. L. Anderson, R. Ding, A. Ellern, and D. W. Armstrong, J. Am. Chem. Soc., 127, 593-604, (2005).

15. Q. Liu, F. van Rantwijk and R. A. Sheldon, J. Chem. Technol. Biotechnol., 81, 401-405 (2006).

16. Z.X. Zhang, H.Y. Zhou, L. Yang, K. Tachibana, K. Kamijima and J. Xu, Electrochimica Acta., 53, 4833-4838, (2008).

17. H. L. Ngo, K. LeCompte, L. Hargens and A. B. McEwen, Thermochim. Acta, 357, 97 - 102, (2000)

18. K. Huang, X. Han, X., Zhang and D.W. Armstrong, Anal. Bioanal Chem, 389, 2265-2275, (2007)

19. C. Jin, C. Ye, B.S.Philips, J.S. Zabinski, X. Liu, W. Liu and J.M. Shreeve, J. Mater. Chem., 16, 1529-1535 (2006) 